\title{
Existence and multiplicity of solutions for discrete Neumann-Steklov problems with singular $\phi$-Laplacian
}

\author{
Yanqiong $\mathrm{Lu}^{1 *}$, Ruyun $\mathrm{Ma}^{1}$ and Bo $\mathrm{Lu}^{2}$
}

\section{"Correspondence:} luyq8610@126.com

'Department of Mathematics, Northwest Normal University, Lanzhou, 730070, P.R. China Full list of author information is available at the end of the article

\begin{abstract}
This paper establishes the existence and multiplicity of solutions for the discrete Neumann-Steklov problem with singular $\phi$-Laplacian by using the method of lower and upper solutions, a priori estimates and Brouwer degree theory.
\end{abstract}

MSC: 34B15; 39A 12

Keywords: Neumann-Steklov conditions; discrete; singular $\phi$-Laplacian; Brouwer degree

\section{Introduction}

Recently, the various existence results for quasilinear equation problems

$$
\left(\phi\left(u^{\prime}\right)\right)^{\prime}+f\left(t, u, u^{\prime}\right)=0, \quad t \in(0, T)
$$

and the discrete form

$$
\nabla\left(\phi\left(\Delta u_{k}\right)\right)+f\left(t_{k}, u_{k}, \Delta u_{k}\right)=0, \quad k \in\{2,3, \ldots, N-1\}=:[2, N-1]_{\mathbb{Z}}
$$

subjected to Dirichlet, periodic or Neumann boundary conditions on $[0, T]$ or $[2, N-1]_{\mathbb{N}}$ have been studied by many authors such as Mawhin, Bereanu, Jebelean, Torres, Thompson, Coelho, Corsato, Obersnel, Omari, Rivetti, Ma and so on [1-9]. Here $\phi:(-a, a) \rightarrow \mathbb{R}$ is an increasing homeomorphism with $\phi(0)=0$, the model example is

$$
\phi(s)=\frac{s}{\sqrt{1-\kappa s^{2}}}, \quad s \in\left(-\frac{1}{\sqrt{\kappa}}, \frac{1}{\sqrt{\kappa}}\right), \kappa>0 \text { is a constant. }
$$

$\Delta$ is a forward difference operator with $u_{k}=u\left(t_{k}\right), \Delta u_{k}=u\left(t_{k+1}\right)-u\left(t_{k}\right), t_{N}=T$ and $\nabla$ is a backward difference operator with $\nabla u_{k}=u\left(t_{k}\right)-u\left(t_{k-1}\right), t_{1}=0, f:[0, T] \times \mathbb{R}^{2} \rightarrow \mathbb{R}$ is continuous. In addition, the nonlinear difference equations play an important role in many fields such as biology, engineering, science and technology where discrete phenomena abound, meanwhile, from the advent and rise of computers, differential equations have been solved by employing their approximative difference equations formulations, e.g., see [7-18] and the references therein.

\section{Springer}


This paper focuses on the existence and multiplicity of solutions for the discrete Neumann-Steklov problem with singular $\phi$-Laplacian operator

$$
\begin{aligned}
& \nabla\left(\phi\left(\Delta u_{k}\right)\right)+f\left(k, u_{k}, \Delta u_{k}\right)=0, \quad k \in[2, N-1]_{\mathbb{Z}}, \\
& \phi\left(\Delta u_{1}\right)=h_{1}\left(u_{1}\right), \quad \phi\left(\Delta u_{N-1}\right)=h_{N}\left(u_{N}\right),
\end{aligned}
$$

where $h_{1}, h_{N} \in C(\mathbb{R}, \mathbb{R})$ and $f:[2, N-1]_{\mathbb{Z}} \times \mathbb{R}^{2} \rightarrow \mathbb{R}$ is continuous with respect to the second and third variables.

Obviously, the Neumann-Steklov conditions of (1.1) can be written in the equivalent classical form

$$
\Delta u_{1}=g_{1}\left(u_{1}\right), \quad \Delta u_{N-1}=g_{N}\left(u_{N}\right)
$$

with $g_{0}: \mathbb{R} \rightarrow(-a, a)$, and $g_{N}: \mathbb{R} \rightarrow(-a, a)$ given by $g_{1}=\phi^{-1} \circ h_{1}, g_{N}=\phi^{-1} \circ h_{N}$. In addition, $u \in \mathbb{R}^{N}$ satisfies $u_{2}=u_{1}+g_{1}\left(u_{1}\right), u_{N-1}=u_{N}-g_{N}\left(u_{N}\right)$. Notice that if $h_{1}, h_{N} \equiv 0$, then problem (1.1) is degenerate to the Neumann problem, which was studied in [7-9]. However, as far as we know, there is very little work on the existence of solutions of difference equation with nonlinear boundary value conditions. Motivated by the above works [2, 7-9], we shall discuss the existence and multiplicity of solutions of (1.1).

The rest of this paper is organized as follows. In Section 2, we state some notations and preliminary results. Section 3 contains the proof of the existence of one solution of (1.1) when the nonlinearity $f$ and $h_{0}, h_{N}$ satisfy some suitable sign conditions. In Section 4 , we extend the classical method of upper and lower solutions to the Neumann-Steklov problem, and we obtain Ambrosetti-Prodi type results for the Neumann-Steklov problem (1.1) in Section 5.

\section{Preliminaries}

For convenience, we list a few notations that will be used throughout this paper. Let $a, b \in$ $\mathbb{N}$ with $a<b$, we denote $[a, b]_{\mathbb{Z}}:=\{a, a+1, \ldots, b\}$. In addition, we denote $\sum_{s=a}^{b} u_{s}=0$ with $b<a$ and $\prod_{s=a}^{b} u_{s}=1$ with $b<a$.

For $\mathbf{u}=\left(u_{1}, \ldots, u_{p}\right) \in \mathbb{R}^{p}$, set $\|\mathbf{u}\|_{\infty}=\max _{1 \leq k \leq p}\left|u_{k}\right|$. If $\boldsymbol{\alpha}, \boldsymbol{\beta} \in \mathbb{R}^{p}$, we write $\boldsymbol{\alpha} \leq \boldsymbol{\beta}$ (respectively $\boldsymbol{\alpha}<\boldsymbol{\beta}$ ) if $\alpha_{k} \leq \beta_{k}$ (resp. $\alpha_{k}<\beta_{k}$ ) for all $1 \leq k \leq p$. The following assumption upon $\phi$ (called singular) is made throughout the paper:

$\left(H_{\phi}\right) \phi:(-a, a) \rightarrow \mathbb{R}(0<a<\infty)$ is an increasing homeomorphism with $\phi(0)=0$.

Let $N \in \mathbb{N}$ with $N \geq 4$ be fixed and $\mathbf{u}=\left(u_{1}, u_{2}, \ldots, u_{N}\right) \in \mathbb{R}^{N}$. Then we denote

$$
\Delta \mathbf{u}=\left(\Delta u_{1}, \ldots, \Delta u_{N-1}\right) \in \mathbb{R}^{N-1}
$$

by $\Delta u_{k}=u_{k+1}-u_{k}$ for $k \in[1, N-1]_{\mathbb{Z}},\|\Delta \mathbf{u}\|_{\infty}:=\max _{k \in[1, N-1]_{\mathbb{Z}}}\left|\Delta u_{k}\right|$ and $\|\mathbf{u}\|=\|\mathbf{u}\|_{\infty}+$ $\|\Delta \mathbf{u}\|_{\infty} ;$ if $\|\Delta \mathbf{u}\|_{\infty}<a$, then we define

$$
\nabla(\phi(\Delta \mathbf{u}))=\left(\nabla\left(\phi\left(\Delta u_{2}\right)\right), \ldots, \nabla\left(\phi\left(\Delta u_{N-1}\right)\right)\right) \in \mathbb{R}^{N-2}
$$

by $\nabla\left(\phi\left(\Delta u_{k}\right)\right)=\phi\left(\Delta u_{k}\right)-\phi\left(\Delta u_{k-1}\right)$ for $k \in[2, N-1]_{\mathbb{Z}}$. 
Let $f:[2, N-1]_{\mathbb{Z}} \times \mathbb{R}^{2} \rightarrow \mathbb{R}$ be a continuous function with respect to the second and third variables. Then its Nemytskii operator $N_{f}(\mathbf{u}): \mathbb{R}^{N} \rightarrow \mathbb{R}^{N-2}$ is given by

$$
N_{f}(\mathbf{u})=\left(f\left(2, u_{2}, \Delta u_{2}\right), \ldots, f\left(N-1, u_{N-1}, \Delta u_{N-1}\right)\right) .
$$

It follows that $N_{f}$ is continuous and takes bounded sets into bounded sets.

Let $P, Q$ be the projectors defined by

$$
P \mathbf{u}=u_{1}, \quad \overline{\mathbf{u}}=Q \mathbf{u}=\frac{1}{N-2} \sum_{k=2}^{N-1} u_{k} \quad \text { for all } \mathbf{u} \in \mathbb{R}^{N}
$$

We also define the linear mapping

$$
\begin{aligned}
& \tilde{Q}: \mathbb{R}^{N} \times \mathbb{R}^{2} \rightarrow \mathbb{R}^{N} \times \mathbb{R}^{2}, \\
& (\mathbf{u}, A, B)=\left(Q \mathbf{u}-\frac{B-A}{N-2}, 0,0\right) .
\end{aligned}
$$

Moreover,

$$
\begin{aligned}
\tilde{Q}^{2}(\mathbf{u}, A, B) & =\tilde{Q}\left(Q(\mathbf{u})-\frac{B-A}{N-2}, 0,0\right)=\left(Q\left(Q(\mathbf{u})-\frac{B-A}{N-2}\right), 0,0\right) \\
& =\left(Q \mathbf{u}-\frac{B-A}{N-2}, 0,0\right)=\tilde{Q}(\mathbf{u}, A, B),
\end{aligned}
$$

which means $\tilde{Q}$ is also a projector. Let the linear operator $H_{i}: \mathbb{R}^{N} \rightarrow \mathbb{R}^{N-1}$ as follows:

$$
H_{1} \mathbf{u}=\sum_{s=1}^{k-1} u_{s}, \quad H_{2} \mathbf{u}=\sum_{s=2}^{k} u_{s}, \quad k \in[2, N]_{\mathbb{Z}} .
$$

The vector space $\mathbb{R}^{N}$ will be a Banach space endowed with the norm $\|\mathbf{u}\|$. For $\mathbf{u} \in \mathbb{R}^{N}$, we also give some notations as follows:

$$
\mathbf{u}^{+}=\max \{\mathbf{u}, 0\}, \quad \mathbf{u}^{-}=\min \{\mathbf{u}, 0\}, \quad \mathbf{u}_{M}=\max _{1 \leq k \leq N} u_{k}, \quad \mathbf{u}_{L}=\min _{1 \leq k \leq N} u_{k} .
$$

We set $B(\mathbf{0}, \rho):=\left\{\mathbf{u} \in \mathbb{R}^{N} \mid\|\mathbf{u}\|_{\infty}<\rho\right\}(\rho>0)$, and for shortness, we shall write $B_{\rho}$ instead of $B(\mathbf{0}, \rho)$.

Lemma 2.1 Let $f:[2, N-1]_{\mathbb{Z}} \rightarrow \mathbb{R}$ be a function and consider the discrete NeumannSteklov problem

$$
\nabla\left(\phi\left(\Delta u_{k}\right)\right)=f(k), k \in[2, N-1]_{\mathbb{Z}}, \quad \phi\left(\Delta u_{1}\right)=A, \quad \phi\left(\Delta u_{N-1}\right)=B .
$$

A function $\mathbf{u}$ is a solution of (2.2) if and only if

$$
Q f-\frac{B-A}{N-2}=0,
$$


i.e., if and only if

$$
\tilde{Q}(f, A, B)=0,
$$

in which case the solutions of (2.2) are given by the operator equation

$$
\mathbf{u}=P \mathbf{u}+H_{1} \circ \phi^{-1} \circ\left[A+H_{2} f\right] .
$$

Proof Problem (2.2) can be written as

$$
\phi\left(\Delta u_{k}\right)-\phi\left(\Delta u_{k-1}\right)=f(k), \quad \phi\left(\Delta u_{1}\right)=A, \quad \phi\left(\Delta u_{N-1}\right)=B,
$$

which implies that

$$
\Delta u_{k}=\phi^{-1} \circ\left(A+H_{2} f\right)
$$

This together with (2.3) concludes that

$$
\phi\left(\Delta u_{N-1}\right)=A+(N-2) Q f=B .
$$

Moreover, a function $\mathbf{u}$ is a solution of (2.2) if and only if (2.3) holds, i.e., if and only if (2.4) is true.

By summing from $s=1$ to $k$ in (2.6), it follows that

$$
u_{k}-u_{1}=\sum_{s=1}^{k-1} \Delta u_{s}=\sum_{s=1}^{k-1} \phi^{-1} \circ\left(A+H_{2} f\right)=H_{1} \circ \phi^{-1} \circ\left(A+H_{2} f\right) .
$$

Thus, we have that the solutions of (2.2) are given by (2.5).

Remark 2.2 Lemma 2.1 means that $(f, A, B)$ belongs to the range of the nonlinear mapping $\mathbf{u} \rightarrow\left[\nabla(\phi(\Delta \mathbf{u})), \phi\left(\Delta u_{1}\right), \phi\left(\Delta u_{N-1}\right)\right]$ if and only if $\tilde{Q}(f, A, B)=0$.

Lemma 2.3 Let $F: \mathbb{R}^{N} \rightarrow \mathbb{R}^{N-2}$ be a continuous operator which takes bounded sets into bounded sets, and consider the abstract discrete Neumann-Steklov problem

$$
\nabla(\phi(\Delta \mathbf{u}))=F(\mathbf{u}), \quad \phi\left(\Delta u_{1}\right)=h_{1}\left(u_{1}\right), \quad \phi\left(\Delta u_{N-1}\right)=h_{N}\left(u_{N}\right) .
$$

A function $\mathbf{u}$ is a solution of (2.7) if and only if $\mathbf{u} \in \mathbb{R}^{N}$ is a fixed point of the continuous operator $\mathcal{A}_{F}: \mathbb{R}^{N} \rightarrow \mathbb{R}^{N}$ defined by $\mathcal{A}_{F}(\mathbf{u}):=P \mathbf{u}+Q F(\mathbf{u})-\frac{1}{N-2}\left[h_{N}\left(u_{N}\right)-h_{1}\left(u_{1}\right)\right]+H_{1} \circ \phi^{-1} \circ$ $\left[H_{2}(I-Q) F(\mathbf{u})+G(\mathbf{u})\right]$, where $G(\mathbf{u}):=\left(G\left(u_{1}\right), G\left(u_{2}\right), \ldots, G\left(u_{N-1}\right)\right) \in \mathbb{R}^{N-1}$ satisfying

$$
G\left(u_{k}\right)=\left(1-\frac{k-1}{N-2}\right) h_{1}\left(u_{1}\right)+\frac{k-1}{N-2} h_{N}\left(u_{N}\right), \quad k \in[1, N-1]_{\mathbb{Z}} .
$$

Furthermore, $\left\|\Delta\left(\mathcal{A}_{F}(\mathbf{u})\right)\right\|_{\infty}<$ a for all $\mathbf{u} \in \mathbb{R}^{N}$ and the operator $\mathcal{A}_{F}(\mathbf{u})$ is completely continuous on $\mathbb{R}^{N}$. 
Proof It follows from Lemma 2.1 that problem (2.7) is equivalent to

$$
\begin{aligned}
& \nabla(\phi(\Delta \mathbf{u}))=F(\mathbf{u})-\left[Q F(\mathbf{u})-\frac{h_{N}\left(u_{N}\right)-h_{1}\left(u_{1}\right)}{N-2}\right] \\
& Q F(\mathbf{u})-\frac{h_{N}\left(u_{N}\right)-h_{1}\left(u_{1}\right)}{N-2}=0 .
\end{aligned}
$$

Let $\mathbf{u} \in \mathbb{R}^{N}$, then

$$
\begin{aligned}
Q\left[F(\mathbf{u})-\left[Q F(\mathbf{u})-\frac{h_{N}\left(u_{N}\right)-h_{1}\left(u_{1}\right)}{N-2}\right]\right] & =Q F(\mathbf{u})-Q F(\mathbf{u})+Q\left(\frac{h_{N}\left(u_{N}\right)-h_{1}\left(u_{1}\right)}{N-2}\right) \\
& =\frac{h_{N}\left(u_{N}\right)-h_{1}\left(u_{1}\right)}{N-2} .
\end{aligned}
$$

By Lemma 2.1, the first equation in (2.9) can be rewritten as

$$
\mathbf{u}=P \mathbf{u}+H_{1} \circ \phi^{-1} \circ\left[h_{1}\left(u_{1}\right)+H_{2}\left(F(\mathbf{u})-\left[Q F(\mathbf{u})-\frac{h_{N}\left(u_{N}\right)-h_{1}\left(u_{1}\right)}{N-2}\right]\right)\right] .
$$

This together with the second equation in (2.9) implies that (2.9) can be written as the single equation

$$
\begin{aligned}
\mathbf{u} & -P \mathbf{u}-\left(Q F(\mathbf{u})-\frac{h_{N}\left(u_{N}\right)-h_{1}\left(u_{1}\right)}{N-2}\right) \\
& -H_{1} \circ \phi^{-1} \circ\left[h_{1}\left(u_{1}\right)+H_{2}\left(F(\mathbf{u})-\left[Q F(\mathbf{u})-\frac{h_{N}\left(u_{N}\right)-h_{1}\left(u_{1}\right)}{N-2}\right]\right)\right]=0,
\end{aligned}
$$

that is,

$$
\mathbf{u}-P \mathbf{u}-\left(Q F(\mathbf{u})-\frac{h_{N}\left(u_{N}\right)-h_{1}\left(u_{1}\right)}{N-2}\right)-H_{1} \circ \phi^{-1} \circ\left[H_{2}(I-Q) F(\mathbf{u})+G(\mathbf{u})\right]=0
$$

\section{The existence of solutions for nonlinear Neumann-Steklov problems}

In this section, we will show the existence of solutions for the nonlinear Neumann-Steklov problems

$$
\begin{aligned}
& \nabla\left(\phi\left(\Delta u_{k}\right)\right)=f\left(k, u_{k}, \Delta u_{k}\right), \quad k \in[2, N-1]_{\mathbb{Z}}, \\
& \phi\left(\Delta u_{1}\right)=h_{1}\left(u_{1}\right), \quad \phi\left(\Delta u_{N-1}\right)=h_{N}\left(u_{N}\right),
\end{aligned}
$$

where $f:[2, N-1]_{\mathbb{Z}} \times \mathbb{R}^{2} \rightarrow \mathbb{R}$ is continuous with respect to the second and third variables, $h_{1}: \mathbb{R} \rightarrow \mathbb{R}, h_{N}: \mathbb{R} \rightarrow \mathbb{R}$ are continuous.

Clearly, the following Neumann-Steklov problem

$$
\nabla\left(\phi\left(\Delta u_{k}\right)\right)=0, \quad k \in[2, N-1]_{\mathbb{Z}}, \quad \phi\left(\Delta u_{1}\right)=0, \quad \phi\left(\Delta u_{N-1}\right)=1
$$

has no solution, which means that the existence of Neumann-Steklov problem is nontrivial. We will show that some rather general sign conditions upon $f, h_{1}, h_{N}$ suffice to get existence. 
To this end, for $\lambda \in[0,1]$, we introduce the family of abstract nonlinear NeumannSteklov problems

$$
\begin{aligned}
& {\left[\nabla(\phi(\Delta \mathbf{u})), \phi\left(\Delta u_{1}\right), \phi\left(\Delta u_{N-1}\right)\right]} \\
& \quad=\lambda\left[N_{f}(\mathbf{u}), h_{1}\left(u_{1}\right), h_{N}\left(u_{N}\right)\right]+(1-\lambda) \tilde{Q}\left[N_{f}(\mathbf{u}), h_{1}\left(u_{1}\right), h_{N}\left(u_{N}\right)\right],
\end{aligned}
$$

where $\tilde{Q}$ is defined in (2.1), (3.2) can be rewritten in a more explicit form

$$
\begin{aligned}
& \nabla(\phi(\Delta \mathbf{u}))=\lambda N_{f}(\mathbf{u})+(1-\lambda)\left[Q N_{f}(\mathbf{u})-\frac{h_{N}\left(u_{N}\right)-h_{1}\left(u_{1}\right)}{N-2}\right], \\
& \phi\left(\Delta u_{1}\right)=\lambda h_{1}\left(u_{1}\right), \\
& \phi\left(\Delta u_{N-1}\right)=\lambda h_{N}\left(u_{N}\right) .
\end{aligned}
$$

It is worthwhile pointing out that (3.2) coincide with (3.1) for $\lambda=1$, and if $\mathbf{u}$ is a solution of (3.2), then, using the definition of $\tilde{Q}$ and Remark 2.2 to (3.2), we get that

$$
\begin{aligned}
0 & =\lambda \tilde{Q}\left[N_{f}(\mathbf{u}), h_{1}\left(u_{1}\right), h_{N}\left(u_{N}\right)\right]+(1-\lambda) \tilde{Q}\left[N_{f}(\mathbf{u}), h_{1}\left(u_{1}\right), h_{N}\left(u_{N}\right)\right], \\
& =\tilde{Q}\left[N_{f}(\mathbf{u}), h_{1}\left(u_{1}\right), h_{N}\left(u_{N}\right)\right] .
\end{aligned}
$$

Therefore, (3.2) is equivalent to

$$
\begin{aligned}
& {\left[\nabla(\phi(\Delta \mathbf{u})), \phi\left(\Delta u_{1}\right), \phi\left(\Delta u_{N-1}\right)\right]=\lambda\left[N_{f}(\mathbf{u}), h_{1}\left(u_{1}\right), h_{N}\left(u_{N}\right)\right],} \\
& \tilde{Q}\left[N_{f}(\mathbf{u}), h_{1}\left(u_{1}\right), h_{N}\left(u_{N}\right)\right]=0,
\end{aligned}
$$

or, in a more explicit form,

$$
\begin{aligned}
& \nabla\left(\phi\left(\Delta u_{k}\right)\right)=\lambda f\left(k, u_{k}, \Delta u_{k}\right), \quad \phi\left(\Delta u_{1}\right)=\lambda h_{1}\left(u_{1}\right), \quad \phi\left(\Delta u_{N-1}\right)=\lambda h_{N}\left(u_{N}\right), \\
& Q N_{f}(\mathbf{u})-\frac{h_{N}\left(u_{N}\right)-h_{1}\left(u_{1}\right)}{N-2}=0 .
\end{aligned}
$$

For any $\lambda \in[0,1]$, the nonlinear operator $\mathcal{A}$ on $\mathbb{R}^{N}$ associated to (3.2) by Lemma 2.3 is the operator $\mathcal{A}(\lambda, \cdot)$, where $\mathcal{A}$ is defined on $[0,1] \times \mathbb{R}^{N}$ as follows:

$$
\begin{aligned}
\mathcal{A}(\lambda, \mathbf{u})= & P \mathbf{u}+Q\left[\lambda N_{f}(\mathbf{u})+(1-\lambda) Q N_{f}(\mathbf{u})-(1-\lambda) \frac{h_{N}\left(u_{N}\right)-h_{1}\left(u_{1}\right)}{N-2}\right] \\
& -\frac{\lambda h_{N}\left(u_{N}\right)-\lambda h_{1}\left(u_{1}\right)}{N-2}+H_{1} \circ \phi^{-1} \circ\left[H _ { 2 } ( I - Q ) \left[\lambda N_{f}(\mathbf{u})+(1-\lambda) Q N_{f}(\mathbf{u})\right.\right. \\
& \left.\left.-(1-\lambda) \frac{h_{N}\left(u_{N}\right)-h_{1}\left(u_{1}\right)}{N-2}\right]+\lambda G(\mathbf{u})\right] \\
= & P \mathbf{u}+Q N_{f}(\mathbf{u})-\frac{h_{N}\left(u_{N}\right)-h_{1}\left(u_{1}\right)}{N-2} \\
& +H_{1} \circ \phi^{-1} \circ\left[\lambda H_{2}(I-Q) N_{f}(\mathbf{u})+\lambda G(\mathbf{u})\right],
\end{aligned}
$$

where $G$ is defined by (2.8). Clearly, $\mathcal{A}$ is a continuous operator. Moreover, it is not difficult to verify that $\mathcal{A}:[0,1] \times \mathbb{R}^{N} \rightarrow \mathbb{R}^{N}$ is completely continuous.

Now, we give the following technical results to obtain the main result. 
Lemma 3.1 Suppose that there exists $R>0$ such that when $\mathbf{u}_{L} \geq R,\|\Delta \mathbf{u}\|_{\infty}<a$, and when $\mathbf{u}_{M} \leq-R,\|\Delta \mathbf{u}\|_{\infty}<a$, it follows that

$$
\sum_{k=2}^{N-1} f\left(k, u_{k}, \Delta u_{k}\right)-\left[h_{N}\left(u_{N}\right)-h_{1}\left(u_{1}\right)\right] \neq 0 .
$$

If $(\lambda, \mathbf{u}) \in[0,1] \times \mathbb{R}^{N}$ is such that $\mathbf{u}=\mathcal{A}(\lambda, \mathbf{u})$, then $\|\mathbf{u}\|<R+a N$.

Proof Let $(\lambda, \mathbf{u}) \in[0,1] \times \mathbb{R}^{N}$ be such that $\mathbf{u}=\mathcal{A}(\lambda, \mathbf{u})$. Choosing $\lambda=0$, we get

$$
Q N_{f}(\mathbf{u})-\frac{h_{N}\left(u_{N}\right)-h_{1}\left(u_{1}\right)}{N-2}=0 .
$$

This means that

$$
\sum_{k=2}^{N-1} f\left(k, u_{k}, \Delta u_{k}\right)-h_{N}\left(u_{N}\right)+h_{1}\left(u_{1}\right)=0 .
$$

Since

$$
\Delta \mathbf{u}=\Delta(\mathcal{A}(\lambda, \mathbf{u}))=\phi^{-1} \circ\left[\lambda H_{2}(I-Q) N_{f}(\mathbf{u})+\lambda G(\mathbf{u})\right]
$$

it yields that

$$
\|\Delta \mathbf{u}\|_{\infty}<a
$$

If $\mathbf{u}_{M} \leq-R$ (resp. $\mathbf{u}_{L} \geq R$ ), then it follows from (3.6), (3.8) that

$$
\sum_{k=2}^{N-1} f\left(k, u_{k}, \Delta u_{k}\right)-\left[h_{N}\left(u_{N}\right)-h_{1}\left(u_{1}\right)\right] \neq 0 .
$$

By using (3.7), we conclude that

$$
\mathbf{u}_{M}>-R \text { and } \mathbf{u}_{L}<R
$$

Obviously, we get $\mathbf{u}_{M} \leq \mathbf{u}_{L}+\sum_{k=1}^{N-1}\left|\Delta u_{k}\right|$, which together with (3.8), (3.9) leads to

$$
-(R+a(N-1))<u_{L} \leq u_{M} \leq R+a(N-1) .
$$

Thus, (3.8) and (3.10) conclude that $\|\mathbf{u}\|<R+a N$.

Lemma 3.2 Suppose that there exist $R>0$ and $\varepsilon \in\{-1,1\}$ such that when $\mathbf{u}_{L} \geq R,\|\Delta \mathbf{u}\|_{\infty}<$ a, it follows that

$$
\varepsilon\left[\sum_{k=2}^{N-1} f\left(k, u_{k}, \Delta u_{k}\right)-\left[h_{N}\left(u_{N}\right)-h_{1}\left(u_{1}\right)\right]\right]>0 ;
$$


and when $\mathbf{u}_{M} \leq-R,\|\Delta \mathbf{u}\|_{\infty}<$ a, it yields that

$$
\varepsilon\left[\sum_{k=2}^{N-1} f\left(k, u_{k}, \Delta u_{k}\right)-\left[h_{N}\left(u_{N}\right)-h_{1}\left(u_{1}\right)\right]\right]<0 .
$$

Then, for all sufficiently large $\rho>0$,

$$
\operatorname{deg}\left(I-\mathcal{A}(1, \cdot), B_{\rho}, 0\right)=-\varepsilon,
$$

and problem (3.1) has at least one solution.

Proof Let $\rho>R+a N$ be a constant, and let $\mathcal{A}$ be the operator given by (3.5). By using Lemma 3.1 and the homotopy invariance of the Brouwer degree [19], we deduce that

$$
\operatorname{deg}\left(I-\mathcal{A}(0, \cdot), B_{\rho}, 0\right)=\operatorname{deg}\left(I-\mathcal{A}(1, \cdot), B_{\rho}, 0\right) .
$$

On the other hand, we get that

$$
\operatorname{deg}\left(I-\mathcal{A}(0, \cdot), B_{\rho}, 0\right)=\operatorname{deg}\left(I-\left(P+Q N_{f}-\frac{h_{N}\left(u_{N}\right)-h_{1}\left(u_{1}\right)}{N-2}\right), B_{\rho}, 0\right) .
$$

However, the range of the mapping $\mathbf{u} \rightarrow P \mathbf{u}+Q N_{f}(\mathbf{u})-\frac{h_{N}\left(u_{N}\right)-h_{1}\left(u_{1}\right)}{N-2}$ is contained in the subspace of constant functions, isomorphic to $\mathbb{R}$, hence, applying a reduction property of Brouwer degree, we get

$$
\begin{aligned}
& \operatorname{deg}\left(I-\left(P+Q N_{f}-\frac{h_{N}\left(u_{N}\right)-h_{1}\left(u_{1}\right)}{N-2}\right), B_{\rho}, 0\right) \\
& \quad=\operatorname{deg}\left(I-\left.\left(P+Q N_{f}-\frac{h_{N}\left(u_{N}\right)-h_{1}\left(u_{1}\right)}{N-2}\right)\right|_{\mathbb{R}},(-\rho, \rho), 0\right) \\
& \quad=\operatorname{deg}\left(-Q N_{f}+\left.\frac{h_{N}\left(u_{N}\right)-h_{1}\left(u_{1}\right)}{N-2}\right|_{\mathbb{R}},(-\rho, \rho), 0\right) \\
& \quad=\frac{1}{2} \operatorname{sign}\left[-Q N_{f}(\rho)+\frac{h_{N}\left(u_{N}\right)-h_{1}\left(u_{1}\right)}{N-2}\right]-\frac{1}{2} \operatorname{sign}\left[-Q N_{f}(-\rho)+\frac{h_{N}\left(u_{N}\right)-h_{1}\left(u_{1}\right)}{N-2}\right] .
\end{aligned}
$$

However, it follows from (3.11), (3.12) and $\rho>R$ that

$$
Q N_{f}( \pm \rho)-\frac{h_{N}\left(u_{N}\right)-h_{1}\left(u_{1}\right)}{N-2}=\frac{1}{N-2} \sum_{k=2}^{N-1} f(k, \pm \rho, 0)-\frac{h_{N}\left(u_{N}\right)-h_{1}\left(u_{1}\right)}{N-2}
$$

have opposite sign, and using (3.11), (3.12), we deduce that

$$
\operatorname{deg}\left(I-\mathcal{A}(0, \cdot), B_{\rho}, 0\right)=-\varepsilon
$$

Thus, $\operatorname{deg}\left(I-\mathcal{A}(1, \cdot), B_{\rho}, 0\right)=-\varepsilon$, that is to say, there exists $\mathbf{u} \in B_{\rho}$ such that $\mathbf{u}=\mathcal{A}(1, \mathbf{u})$, which is a solution of problem (3.1). 
Lemma 3.2 may imply the existence of a positive solution of (3.1). A limiting argument allows to weaken the sign condition; however, this generalization can also be proved directly using another way based on the following lemma, see Section 4.

For any $\mathbf{u} \in \mathbb{R}^{N}$, decompose it in the form

$$
\mathbf{u}=\bar{u}+\tilde{\mathbf{u}}, \quad \text { here } \bar{u}=u_{1}, \tilde{u}_{1}=0,
$$

and let $W=\left\{\mathbf{u} \in \mathbb{R}^{N} \mid u_{1}=0\right\}$.

Lemma 3.3 The set $\mathcal{S}$ of the solutions $(\bar{u}, \tilde{\mathbf{u}}) \in \mathbb{R} \times W$ of the following problem

$$
\begin{gathered}
\begin{aligned}
\nabla\left(\phi\left(\Delta \tilde{u}_{k}\right)\right)= & f\left(k, \bar{u}+\tilde{u}_{k}, \Delta \tilde{u}_{k}\right)-\frac{1}{N-2} \sum_{s=2}^{N-1} f\left(s, \bar{u}+\tilde{u}_{s}, \Delta \tilde{u}_{s}\right) \\
& +\frac{h_{N}\left(\bar{u}+\tilde{u}_{N}\right)-h_{1}(\bar{u})}{N-2},
\end{aligned} \\
\phi\left(\Delta \tilde{u}_{1}\right)=h_{1}(\bar{u}), \quad \phi\left(\Delta \tilde{u}_{N-1}\right)=h_{N}\left(\bar{u}+\tilde{u}_{N}\right)
\end{gathered}
$$

contain a continuum $\mathcal{C}$ whose projection on $\mathbb{R}$ is $\mathbb{R}$ and whose projection on $W$ is contained in the ball $B_{a N}$.

Proof From Lemma 2.3 and (3.15), for any fixed $\bar{u} \in \mathbb{R}$, problem (3.16) is equivalent to the fixed point problem in $\mathbb{R}^{N}$ as follows:

$$
\tilde{\mathbf{u}}=H_{1} \circ \phi^{-1} \circ\left[H_{2}(I-Q) N_{f}(\bar{u}+\tilde{\mathbf{u}})+G(\bar{u}+\tilde{\mathbf{u}})\right]=: \tilde{\mathcal{A}}(\bar{u}, \tilde{\mathbf{u}}) .
$$

It is easy to see that $\tilde{\mathcal{A}}$ is completely continuous in $\mathbb{R}^{N}$, and for each $(\bar{u}, \tilde{\mathbf{u}}) \in \mathbb{R} \times \mathbb{R}^{N-1}$, we get

$$
\|\tilde{\mathcal{A}}(\bar{u}, \tilde{\mathbf{u}})\|=\|\tilde{\mathcal{A}}(\bar{u}, \tilde{\mathbf{u}})\|_{\infty}+\|\Delta(\tilde{\mathcal{A}}(\bar{u}, \tilde{\mathbf{u}}))\|_{\infty}<a N .
$$

Moreover, for any fixed $\bar{u} \in \mathbb{R}$, any possible fixed point $\tilde{\mathbf{u}}$ of $\tilde{\mathcal{A}}(\bar{u}, \cdot)$ satisfies

$$
\|\tilde{\mathbf{u}}\|<a N
$$

On the other hand, by the same reasons, for any fixed $\lambda \in[0,1]$, each possible fixed point $\tilde{\mathbf{u}}$ of

$$
\tilde{\mathcal{A}}(\lambda, 0, \cdot):=H_{1} \circ \phi^{-1} \circ\left[\lambda H_{2}(I-Q) N_{f}(\cdot)+\lambda G(\cdot)\right]
$$

satisfies (3.17), which yields that

$$
\begin{aligned}
\operatorname{deg}\left(I-\tilde{\mathcal{A}}(0, \cdot), B_{a N}, 0\right) & =\operatorname{deg}\left(I-\tilde{\mathcal{A}}(1,0, \cdot), B_{a N}, 0\right) \\
& =\operatorname{deg}\left(I-\tilde{\mathcal{A}}(0,0, \cdot), B_{a N}, 0\right)=\operatorname{deg}\left(I, B_{a N}, 0\right)=1 .
\end{aligned}
$$

Therefore, from (3.18), (3.19) and [8], Lemma 5, there exists a continuum $\mathcal{C}$ whose projection on $\mathbb{R}$ is $\mathbb{R}$ and whose projection on $W$ is contained in the ball $B_{a N}$. 
Theorem 3.4 Suppose that there exist $R>0$ and $\varepsilon \in\{-1,1\}$ such that when $\mathbf{u}_{L} \geq R$, $\|\Delta \mathbf{u}\|_{\infty}<a$, it follows that

$$
\varepsilon\left[\sum_{k=1}^{N} f\left(k, u_{k}, \Delta u_{k}\right)-\left[h_{N}\left(u_{N}\right)-h_{1}\left(u_{1}\right)\right]\right] \geq 0
$$

and when $\mathbf{u}_{M} \leq-R,\|\Delta \mathbf{u}\|_{\infty}<$ a, it yields that

$$
\varepsilon\left[\sum_{k=1}^{N} f\left(k, u_{k}, \Delta u_{k}\right)-\left[h_{N}\left(u_{N}\right)-h_{1}\left(u_{1}\right)\right]\right] \leq 0
$$

Then problem (3.1) has at least one solution.

Proof From Lemma 3.3, let us consider the continuum $\mathcal{C}$. If $(R+a N, \tilde{\mathbf{u}}) \in \mathcal{C}$, then $R+a N+$ $\tilde{u}>R$ and it follows from (3.20) that

$$
\varepsilon\left[\sum_{k=1}^{N} f\left(k, R+a N+\tilde{u}_{k}, \Delta \tilde{u}_{k}\right)-\left[h_{N}\left(u_{N}\right)-h_{1}\left(u_{1}\right)\right]\right] \geq 0 .
$$

If $(-R-a N, \tilde{\mathbf{u}}) \in \mathcal{C}$, then $-R-a N+\tilde{u}<-R$ and it follows from (3.21) that

$$
\varepsilon\left[\sum_{k=1}^{N} f\left(k,-R-a N+\tilde{u}_{k}, \Delta \tilde{u}_{k}\right)-\left[h_{N}\left(u_{N}\right)-h_{1}\left(u_{1}\right)\right]\right] \leq 0 .
$$

From the intermediate value theorem for a continuous function on a connected set, there exists $(\bar{u}, \tilde{\mathbf{u}}) \in \mathcal{C}$ such that

$$
\varepsilon\left[\sum_{k=1}^{N} f\left(k, \bar{u}+\tilde{u}_{k}, \Delta \tilde{u}_{k}\right)-\left[h_{N}\left(u_{N}\right)-h_{1}\left(u_{1}\right)\right]\right]=0,
$$

which means that $\mathbf{u}=\bar{u}+\tilde{\mathbf{u}}$ is a solution of (3.1).

Corollary 3.5 Letg $:[2, N-1]_{\mathbb{Z}} \times \mathbb{R}^{2} \rightarrow \mathbb{R}, p:[2, N-1]_{\mathbb{Z}} \times \mathbb{R} \rightarrow \mathbb{R}, h_{1}: \mathbb{R} \rightarrow \mathbb{R}, h_{N}: \mathbb{R} \rightarrow \mathbb{R}$ be continuous, which satisfies $g$ is bounded on $[2, N-1]_{\mathbb{Z}} \times \mathbb{R} \times(-a, a)$ and $h_{1}, h_{N}$ are bounded on $\mathbb{R}$. If

$$
\begin{aligned}
& \lim _{u \rightarrow-\infty}\left[p(k, u)-\frac{h_{N}\left(u_{N}\right)-h_{1}\left(u_{1}\right)}{N-2}\right]=+\infty, \\
& \lim _{u \rightarrow+\infty}\left[p(k, u)-\frac{h_{N}\left(u_{N}\right)-h_{1}\left(u_{1}\right)}{N-2}\right]=-\infty,
\end{aligned}
$$

or

$$
\begin{aligned}
& \lim _{u \rightarrow-\infty}\left[p(k, u)-\frac{h_{N}\left(u_{N}\right)-h_{1}\left(u_{1}\right)}{N-2}\right]=-\infty \\
& \lim _{u \rightarrow+\infty}\left[p(k, u)-\frac{h_{N}\left(u_{N}\right)-h_{1}\left(u_{1}\right)}{N-2}\right]=+\infty
\end{aligned}
$$


uniformly in $k \in[2, N-1]_{\mathbb{Z}}$, then the problem

$$
\begin{aligned}
& \nabla\left(\phi\left(\Delta u_{k}\right)\right)+p\left(k, u_{k}\right)=g\left(k, u_{k}, \Delta u_{k}\right), \quad k \in[2, N-1]_{\mathbb{Z}}, \\
& \phi\left(\Delta u_{1}\right)=h_{1}\left(u_{1}\right), \quad \phi\left(\Delta u_{N-1}\right)=h_{N}\left(u_{N}\right)
\end{aligned}
$$

has at least one solution.

Corollary 3.6 Let $g:[2, N-1]_{\mathbb{Z}} \times \mathbb{R}^{2} \rightarrow \mathbb{R}$ be continuous and bounded on $[2, N-1]_{\mathbb{Z}} \times$ $\mathbb{R} \times(-a, a), h_{1}: \mathbb{R} \rightarrow \mathbb{R}, h_{N}: \mathbb{R} \rightarrow \mathbb{R}$ be continuous and satisfy

$$
\lim _{|s| \rightarrow \infty} \frac{h_{1}(s)}{s}=0, \quad \lim _{|s| \rightarrow \infty} \frac{h_{N}(s)}{s}=0 .
$$

Then, for any fixed $\mu \neq 0$, the problem

$$
\begin{aligned}
& \nabla\left(\phi\left(\Delta u_{k}\right)\right)+\mu u_{k}=g\left(k, u_{k}, \Delta u_{k}\right), \quad k \in[2, N-1]_{\mathbb{Z}}, \\
& \phi\left(\Delta u_{1}\right)=h_{1}\left(u_{1}\right), \quad \phi\left(\Delta u_{N-1}\right)=h_{N}\left(u_{N}\right)
\end{aligned}
$$

has at least one solution.

Example 3.7 Let $\mathbf{e}=\left(e_{2}, e_{3}, \ldots, e_{N-1}\right) \in \mathbb{R}^{N-2}, m \in \mathbb{R}, n \in \mathbb{R} \backslash\{0\}, p>1$ and $q \geq 0$. Then the problem

$$
\begin{aligned}
& \nabla\left(\phi\left(\Delta u_{k}\right)\right)+m\left|\Delta u_{k}\right|^{q}+n\left|u_{k}\right|^{p-2} u_{k}=e_{k}, \quad k \in[2, N-1]_{\mathbb{Z}} \\
& \phi\left(\Delta u_{1}\right)=h_{1}\left(u_{1}\right), \quad \phi\left(\Delta u_{N-1}\right)=h_{N}\left(u_{N}\right)
\end{aligned}
$$

has at least one solution for each continuous bounded function $h_{1}, h_{N}: \mathbb{R} \rightarrow \mathbb{R}$, where $\phi(s)=\frac{s}{\sqrt{1-\kappa s^{2}}}$ and $\kappa>0$ is constant.

\section{Upper and lower solutions for the nonlinear Neumann-Steklov problem}

In this section, we give the method of upper and lower solutions to the nonlinear Neumann-Steklov boundary value problem

$$
\begin{aligned}
& \nabla\left(\phi\left(\Delta u_{k}\right)\right)=f\left(k, u_{k}, \Delta u_{k}\right), \quad k \in[2, N-1]_{\mathbb{Z}}, \\
& \phi\left(\Delta u_{1}\right)=h_{1}\left(u_{1}\right), \quad \phi\left(\Delta u_{N-1}\right)=h_{N}\left(u_{N}\right),
\end{aligned}
$$

which extends the method of upper and lower solutions for the linear Neumann boundary value problem [8], Theorem 3, Theorem 4 and Remark 8.

Definition 4.1 A function $\boldsymbol{\alpha}=\left(\alpha_{1}, \ldots, \alpha_{N}\right)\left(\right.$ resp. $\left.\boldsymbol{\beta}=\left(\beta_{1}, \ldots, \beta_{N}\right)\right)$ is called a lower solution (resp. an upper solution) for (4.1) if $\|\Delta \alpha\|_{\infty}<a$ (resp. $\|\Delta \beta\|_{\infty}<a$ ) and

$$
\begin{aligned}
& \nabla\left(\phi\left(\Delta \alpha_{k}\right)\right) \geq f\left(k, \alpha_{k}, \Delta \alpha_{k}\right), \quad\left(\text { resp. } \nabla\left(\phi\left(\Delta \beta_{k}\right)\right) \leq f\left(k, \beta_{k}, \Delta \beta_{k}\right)\right) k \in[2, N-1]_{\mathbb{Z}} \\
& \phi\left(\Delta \alpha_{1}\right) \geq h_{1}\left(\alpha_{1}\right), \quad \phi\left(\Delta \alpha_{N-1}\right) \leq h_{N}\left(\alpha_{N}\right) \\
& \quad\left(\text { resp. } \phi\left(\Delta \beta_{1}\right) \leq h_{1}\left(\beta_{1}\right), \phi\left(\Delta \beta_{N-1}\right) \geq h_{N}\left(\beta_{N}\right)\right) .
\end{aligned}
$$

Such a lower (resp. an upper) solution is called strict if inequality (4.2) is strict. 
Theorem 4.2 If (4.1) has a lower solution $\boldsymbol{\alpha}=\left(\alpha_{1}, \alpha_{2}, \ldots, \alpha_{N}\right)$ and an upper solution $\boldsymbol{\beta}=$ $\left(\beta_{1}, \beta_{2}, \ldots, \beta_{N}\right)$ such that $\boldsymbol{\alpha} \leq \boldsymbol{\beta}$, then (4.1) has a solution $\mathbf{u}$ such that $\boldsymbol{\alpha} \leq \mathbf{u} \leq \boldsymbol{\beta}$. Moreover, if $\boldsymbol{\alpha}$ and $\boldsymbol{\beta}$ are strict, then $\boldsymbol{\alpha}<\mathbf{u}<\boldsymbol{\beta}$, and

$$
\operatorname{deg}\left(I-\mathcal{A}_{f}, \Omega_{\boldsymbol{\alpha}, \boldsymbol{\beta}}, \mathbf{0}\right)=-1,
$$

where $\Omega_{\boldsymbol{\alpha}, \boldsymbol{\beta}}=\left\{\mathbf{u} \in \mathbb{R}^{N} \mid \boldsymbol{\alpha}<\mathbf{u}<\boldsymbol{\beta},\|\Delta \mathbf{u}\|_{\infty}<a\right\}$, and $\mathcal{A}_{f}$ is the fixed point operator associated to (4.1).

Proof Let $p:[2, N-1]_{\mathbb{Z}} \times \mathbb{R} \rightarrow \mathbb{R}$ be a continuous function defined by

$$
p(k, s)= \begin{cases}\beta, & \text { if } s>\beta, \\ s, & \text { if } \alpha \leq s \leq \beta, \\ \alpha, & \text { if } s<\alpha,\end{cases}
$$

and define $F:[2, N-1]_{\mathbb{Z}} \times \mathbb{R}^{2} \rightarrow \mathbb{R}$ by $F(k, u, v)=f(k, p(k, u), v)$. Let us consider the modified problem

$$
\begin{aligned}
& \nabla\left(\phi\left(\Delta u_{k}\right)\right)=F\left(k, u_{k}, \Delta u_{k}\right)+u_{k}-p\left(k, u_{k}\right), \quad k \in[2, N-1]_{\mathbb{Z}}, \\
& \phi\left(\Delta u_{1}\right)=h_{1}\left(p\left(1, u_{1}\right)\right)+\arctan \left[u_{1}-p\left(1, u_{1}\right)\right], \\
& \phi\left(\Delta u_{N-1}\right)=h_{N}\left(p\left(N, u_{N}\right)\right)+\arctan \left[-u_{N}+p\left(N, u_{N}\right)\right] .
\end{aligned}
$$

We claim that if $\mathbf{u}$ is a solution of (4.4), then $\boldsymbol{\alpha} \leq \mathbf{u} \leq \boldsymbol{\beta}$, so that $\mathbf{u}$ is a solution of (4.1).

We first prove that $\alpha \leq u$. Suppose on the contrary that there exists $k_{0} \in[1, N]_{\mathbb{Z}}$ such that $\max _{k \in[1, N]_{\mathbb{Z}}}(\boldsymbol{\alpha}-\mathbf{u})=\alpha_{k_{0}}-u_{k_{0}}>0$. If $k_{0} \in[2, N-1]_{\mathbb{Z}}$, then $\Delta \alpha_{k_{0}-1} \geq \Delta u_{k_{0}-1}$ and $\Delta \alpha_{k_{0}} \leq$ $\Delta u_{k_{0}}$. Since $\phi$ is an increasing homeomorphism, we have that $\nabla\left(\phi\left(\Delta \alpha_{k_{0}}\right)\right) \leq \nabla\left(\phi\left(\Delta u_{k_{0}}\right)\right)$. Moreover, it follows from $\alpha$ is a lower solution of (4.1) that

$$
\begin{aligned}
\nabla\left(\phi\left(\Delta \alpha_{k_{0}}\right)\right) & \leq \nabla\left(\phi\left(\Delta u_{k_{0}}\right)\right)=F\left(k_{0}, u_{k_{0}}, \Delta u_{k_{0}}\right)+u_{k_{0}}-p\left(k_{0}, u_{k_{0}}\right) \\
& =f\left(k_{0}, \alpha_{k_{0}}, \Delta \alpha_{k_{0}}\right)+u_{k_{0}}-\alpha_{k_{0}}<f\left(k_{0}, \alpha_{k_{0}}, \Delta \alpha_{k_{0}}\right) \leq \nabla\left(\phi\left(\Delta \alpha_{k_{0}}\right)\right),
\end{aligned}
$$

which is a contradiction. If $\max _{k \in[1, N]_{\mathbb{Z}}}(\boldsymbol{\alpha}-\mathbf{u})=\alpha_{1}-u_{1}>0$, then $\Delta \alpha_{1} \leq \Delta u_{1}$, and hence

$$
\phi\left(\Delta \alpha_{1}\right) \leq \phi\left(\Delta u_{1}\right)=h_{1}\left(p\left(1, u_{1}\right)\right)+\arctan \left[u_{1}-p\left(1, u_{1}\right)\right]=h_{1}\left(\alpha_{1}\right)+\arctan \left[u_{1}-\alpha_{1}\right]<h_{1}\left(\alpha_{1}\right),
$$

which contradicts the definition of a lower solution. If $\max _{k \in[1, N]_{\mathbb{Z}}}(\boldsymbol{\alpha}-\mathbf{u})=\alpha_{N}-u_{N}>0$, then $\Delta \alpha_{N-1} \geq \Delta u_{N-1}$, and we get that

$$
\begin{aligned}
\phi\left(\Delta \alpha_{N-1}\right) & \geq \phi\left(\Delta u_{N-1}\right)=h_{N}\left(p\left(N, u_{N}\right)\right)+\arctan \left[-u_{N}+p\left(N, u_{N}\right)\right] \\
& =h_{N}\left(\alpha_{N}\right)+\arctan \left[-u_{N}+\alpha_{N}\right]>h_{N}\left(\alpha_{N}\right),
\end{aligned}
$$

which contradicts the definition of a lower solution.

Similarly, we also obtain that $u_{k} \leq \beta_{k}, k \in[1, N]_{\mathbb{Z}}$. Notice that if $\alpha, \beta$ are strict, then, by the same reasoning, we get that $\alpha<\mathbf{u}<\beta$ with $\alpha<\beta$. Moreover, from the definition of 
strict lower and upper solution, neither $\alpha$ nor $\beta$ can be a solution of (4.4). So (4.4) has no solution on the boundary of $\Omega_{\alpha, \beta}$.

From Corollary 3.6, we obtain the existence of a solution for (4.4) and the relation Brouwer degree

$$
\operatorname{deg}\left(I-\tilde{\mathcal{A}}, B_{\rho}, \mathbf{0}\right)=-1
$$

for all large enough $\rho>0$, and the fixed point operator $\tilde{\mathcal{A}}$ associated to (4.4). Furthermore, if $\alpha, \beta$ are strict and $\rho>0$ sufficiently large, then it follows from (4.5) and the additivityexcision property of the Brouwer degree[19] that

$$
\operatorname{deg}\left(I-\tilde{\mathcal{A}}, \Omega_{\boldsymbol{\alpha}, \boldsymbol{\beta}}, \mathbf{0}\right)=\operatorname{deg}\left(I-\tilde{\mathcal{A}}, B_{\rho}, \mathbf{0}\right)=-1
$$

It is easy to see that the completely continuous operator $\mathcal{A}_{f}$ with (4.1) is equal to $\tilde{\mathcal{A}}$ on $\bar{\Omega}_{\alpha, \beta}$, which means that $\operatorname{deg}\left(I-\mathcal{A}_{f}, \Omega_{\boldsymbol{\alpha}, \boldsymbol{\beta}}, \mathbf{0}\right)=-1$.

By a similar argument in [8], Theorem 4, we can conclude that the existence result in Theorem 4.2 also is true when the lower and upper solutions are not ordered.

Theorem 4.3 Assume that (4.1) has a lower solution $\boldsymbol{\alpha}$ and an upper solution $\boldsymbol{\beta}$, then (4.1) has at least one solution.

Proof Let the continuum $\mathcal{C}$ be given in Lemma 3.3. Suppose that there exists some $(\bar{u}, \tilde{\mathbf{u}}) \in$ $\mathcal{C}$ such that

$$
\sum_{k=2}^{N-1} f\left(k, \bar{u}+\tilde{u}_{k}, \Delta \tilde{u}_{k}\right)-\left[h_{N}\left(\bar{u}+\tilde{u}_{N}\right)-h_{1}(\bar{u})\right]=0,
$$

then $\bar{u}+\tilde{\mathbf{u}}$ is a solution of (4.1). If

$$
\sum_{k=2}^{N-1} f\left(k, \bar{u}+\tilde{u}_{k}, \Delta \tilde{u}_{k}\right)-\left[h_{N}\left(\bar{u}+\tilde{u}_{N}\right)-h_{1}(\bar{u})\right]>0, \quad \forall(\bar{u}, \tilde{\mathbf{u}}) \in \mathcal{C},
$$

then, for any fixed $(\bar{u}, \tilde{\mathbf{u}}) \in \mathcal{C}, \bar{u}+\tilde{\mathbf{u}}$ is an upper solution for (4.1) by applying (3.16). Moreover, $\left(\alpha_{M}+a N, \tilde{\mathbf{u}}\right) \in \mathcal{C}$ is an upper solution for (4.1) with $\alpha_{M}+a N+\tilde{u}_{k} \geq \alpha_{k}, k \in[1, N]_{\mathbb{Z}}$. That is, the existence of a solution for (4.1) follows from Theorem 4.2. By a similar way, if

$$
\sum_{k=2}^{N-1} f\left(k, \bar{u}+\tilde{u}_{k}, \Delta \tilde{u}_{k}\right)-\left[h_{N}\left(\bar{u}+\tilde{u}_{N}\right)-h_{1}(\bar{u})\right]<0, \quad \forall(\bar{u}, \tilde{\mathbf{u}}) \in \mathcal{C}
$$

then $\left(\beta_{L}-a N, \tilde{\mathbf{u}}\right) \in \mathcal{C}$ is a lower solution for (4.1) with $\beta_{L}-a N+\tilde{u}_{k} \leq \beta_{k}, k \in[1, N]_{\mathbb{Z}}$, which means that (4.1) has at least one solution follows again from Theorem 4.2.

If we choose the constant lower and upper solutions for (4.1) in Theorem 4.2 and Theorem 4.3 , then we get the following simple existence condition. 
Corollary 4.4 Suppose that there exist constants $a$ and $b$ such that

$$
\begin{aligned}
& f(k, a, 0) \leq 0 \leq f(k, b, 0), \quad k \in[2, N-1]_{\mathbb{Z}} \\
& h_{1}(a) \leq 0 \leq h_{1}(b), \quad h_{N}(b) \leq 0 \leq h_{N}(a) .
\end{aligned}
$$

Then problem (4.1) has at least one solution.

Notice that Theorem 4.3 can deal with the case $a=+\infty$, the key point is the following a priori estimation result.

Lemma 4.5 Let $\varphi:(-a, a) \rightarrow \mathbb{R}(a \leq+\infty)$ be an increasing homeomorphism with $\varphi(0)=0$. Set $\left|h_{1}\right|$ is bounded by $M_{1}$ and $\left|h_{N}\right|$ is bounded by $M_{N}$, there exists $q:[2, N-1]_{\mathbb{Z}} \rightarrow \mathbb{R}$ such that

$$
f(k, u, v) \geq q_{k}, \quad \forall(k, u, v) \in[2, N-1]_{\mathbb{Z}} \times \mathbb{R}^{2} .
$$

If $\mathbf{u}$ is a solution of the nonlinear Neumann-Steklov boundary value problem

$$
\begin{aligned}
& \nabla\left(\varphi\left(\Delta u_{k}\right)\right)=f\left(k, u_{k}, \Delta u_{k}\right), \quad k \in[2, N-1]_{\mathbb{Z}}, \\
& \varphi\left(\Delta u_{1}\right)=h_{1}\left(u_{1}\right), \quad \varphi\left(\Delta u_{N-1}\right)=h_{N}\left(u_{N}\right),
\end{aligned}
$$

then $\|\mathbf{u}\|_{\infty} \leq c$, where $c:=\max \left\{\left|\varphi^{-1}\left[ \pm\left(M_{N}+2 M_{1}+2 N\left\|q^{-}\right\|_{\infty}\right)\right]\right|\right\}$.

Proof Assume that $\mathbf{u}$ is a solution of (4.7). Then it follows that

$$
\varphi\left(\Delta u_{k}\right)=\varphi\left(\Delta u_{1}\right)+\sum_{s=2}^{k} f\left(s, u_{s}, \Delta u_{s}\right), \quad k \in[1, N-1]_{\mathbb{Z}}
$$

and

$$
\|\varphi(\Delta \mathbf{u})\|_{\infty} \leq M_{1}+\sum_{s=2}^{N-1}\left|f\left(s, u_{s}, \Delta u_{s}\right)\right|
$$

From (4.6), we have that there is a function $q$ such that $f$ is bounded from below, and

$$
|f(s, u, v)| \leq f(s, u, v)+2 q_{s}^{-}, \quad(s, u, v) \in[2, N-1]_{\mathbb{Z}} \times \mathbb{R}^{2} .
$$

(4.9) together with (4.8) implies that

$$
\|\varphi(\Delta \mathbf{u})\|_{\infty} \leq M_{1}+\sum_{s=2}^{N-1}\left[f(s, u, v)+2 q_{s}^{-}\right] \leq M_{1}+\sum_{s=2}^{N-1} f(s, u, v)+2 N\left\|q^{-}\right\|_{\infty} .
$$

From this inequality and (3.7), the conclusion is true.

Theorem 4.6 Let $\varphi: \mathbb{R} \rightarrow \mathbb{R}$ be an increasing homeomorphism with $\varphi(0)=0$. Suppose that all conditions of Lemma 4.5 hold and problem (4.7) has a lower solution $\boldsymbol{\alpha}$ and an upper solution $\boldsymbol{\beta}$. Then (4.7) has at least one solution. 
Proof Let $c$ be given in Lemma 4.5, $a_{1}=\max \left\{\|\Delta \alpha\|_{\infty},\|\beta\|_{\infty}, c\right\}+1$ and $a=a_{1}+1$. Set $\phi:(-a, a) \rightarrow \mathbb{R}$ be an increasing homeomorphism such that $\phi=\varphi$ on $\left[-a_{1}, a_{1}\right]$. It is easy to verify that $\alpha$ is a lower solution of (4.1) and $\beta$ is an upper solution of (4.1). From Theorem 4.3, (4.1) has a solution $\mathbf{u}$, which is also a solution of (4.7) by Lemma 4.5.

Remark 4.7 This result is new even in the case $\varphi$ is identity operator, i.e., $\varphi=i d_{\mathbb{R}}$.

\section{Ambrosetti-Prodi type results for the nonlinear Neumann-Steklov problem}

In this section, let us consider the following nonlinear Neumann-Steklov boundary value problem:

$$
\begin{aligned}
& \nabla\left(\phi\left(\Delta u_{k}\right)\right)=f\left(k, u_{k}, \Delta u_{k}\right)-s, \quad k \in[2, N-1]_{\mathbb{Z}}, \\
& \phi\left(\Delta u_{1}\right)=h_{1}\left(u_{1}\right), \quad \phi\left(\Delta u_{N-1}\right)=h_{N}\left(u_{N}\right),
\end{aligned}
$$

where $s \in \mathbb{R}, f:[2, N-1]_{\mathbb{Z}} \times \mathbb{R}^{2} \rightarrow \mathbb{R}$ is continuous with respect to the second and third variables and satisfies the coercivity condition

$$
f(k, u, v) \rightarrow-\infty \quad \text { if }|u| \rightarrow \infty \text { uniformly for } k \in[2, N-1]_{\mathbb{Z}}, v \in(-a, a),
$$

$h_{1}, h_{N}: \mathbb{R} \rightarrow \mathbb{R}$ are continuous and satisfy the following conditions:

$$
\begin{aligned}
& h_{1} \text { is bounded from above, } h_{1}(0)=0, h_{1}(s) \leq 0, s \leq 0 ; \\
& h_{N} \text { is bounded from below, } h_{N}(0)=0, h_{N}(s) \geq 0, s \leq 0 .
\end{aligned}
$$

Now we shall obtain the existence and multiplicity of the solutions of (5.1) in terms of the value of the parameter $s$.

Lemma 5.1 Suppose that $f, h_{1}, h_{N}$ satisfy conditions (5.2), (5.3), (5.4), respectively. Then, for each $b \in \mathbb{R}$, there exists $\rho=\rho(b)>0$ such that any possible solution $\mathbf{u}$ of (5.1) with $s \geq b$ belongs to the open ball $B_{\rho}$.

Proof Let $\mathbf{u}$ be a solution of (5.1) and $s \geq b$. Then it follows that $\mathbf{u}$ satisfies

$$
Q N_{f}(\mathbf{u})-\frac{h_{N}\left(u_{N}\right)-h_{1}\left(u_{1}\right)}{N-2}=s .
$$

From conditions (5.2), (5.3) and (5.4), there is a constant $R>0$ such that if $|u| \geq R, k \in$ $[2, N-1]_{\mathbb{Z}}, v \in(-a, a)$,

$$
f(k, u, v)<b+\frac{\inf _{s \in \mathbb{R}} h_{N}(s)-\sup _{s \in \mathbb{R}} h_{1}(s)}{N-2} .
$$

Therefore, if $\mathbf{u}_{L} \geq R,\|\Delta \mathbf{u}\|_{\infty}<a$ or if $\mathbf{u}_{M} \leq-R,\|\Delta \mathbf{u}\|_{\infty}<a$, then

$$
\begin{aligned}
Q N_{f}(\mathbf{u})-\frac{h_{N}\left(u_{N}\right)-h_{1}\left(u_{1}\right)}{N-2}-s & \leq Q N_{f}(\mathbf{u})-\frac{h_{N}\left(u_{N}\right)-h_{1}\left(u_{1}\right)}{N-2}-b \\
& \leq Q N_{f}(\mathbf{u})-\frac{\inf _{s \in \mathbb{R}} h_{N}(s)-\sup _{s \in \mathbb{R}} h_{1}(s)}{N-2}-b<0 .
\end{aligned}
$$

The conclusion follows from Lemma 3.1. 
Theorem 5.2 Suppose that $f, h_{1}, h_{N}$ satisfy conditions (5.2), (5.3), (5.4), respectively. Then there exists $s_{1} \in \mathbb{R}$ such that problem (5.1) has no solution with $s>s_{1}$, at least one solution with $s=s_{1}$, or at least two solutions with $s<s_{1}$.

Proof Let $S_{i}=\{s \in \mathbb{R} \mid$ (5.1) has at least $i$ solutions $\}(i \geq 1)$. We shall divide the proof into five steps to obtain the conclusion.

Step 1. We show that $S_{1} \neq \emptyset$.

Set $s^{\star}<\min _{k \in[2, N-1]_{\mathbb{Z}}} f(k, 0,0)$. From (5.2), there exists $R^{\star}<0$ such that $\max _{k \in[2, N-1]_{\mathbb{Z}}} f(k$, $\left.R^{\star}, 0\right)<s^{\star}$. Hence, $\alpha \equiv R^{\star}$ is a strict lower solution and $\beta \equiv 0$ is a strict upper solution for (5.1) with $s=s^{\star} . s^{\star} \in S_{1}$ follows from Theorem 4.2.

Step 2. We prove that

$$
\text { if } s_{0} \in S_{1} \quad \text { and } \quad s<s_{0}, \quad \text { then } s \in S_{1} \text {. }
$$

Let $\hat{\mathbf{u}}$ be a solution of (5.1) with $s=s_{0}$ and set $s<s_{0}$. Then it is easy to see that $\hat{\mathbf{u}}$ is a strict upper solution for (5.1). Take $R_{0}<\hat{\mathbf{u}}_{L}^{-}$such that $\max _{k \in[2, N-1]} f\left(k, R_{0}, 0\right)<s$ and $\alpha \equiv R_{0}$ is a strict lower solution for (5.1). Hence, $s \in S_{1}$ by using Theorem 4.2.

Step 3. We claim that

$$
s_{1}=\sup S_{1} \quad \text { is finite and } S_{1} \supset\left(-\infty, s_{1}\right)
$$

Set $s \in \mathbb{R}$ and suppose that (5.1) has a solution $\mathbf{u}$. Then $\|\Delta \mathbf{u}\|_{\infty}<a$ and (5.6) hold, which implies that $s \leq c$ with $c=\sup _{[2, N-1]_{\mathbb{Z}} \times \mathbb{R} \times(-a, a)} f-\frac{\inf _{\mathbb{R}} h_{N}-\sup _{\mathbb{R}} h_{1}}{N-2}$. That is, $s_{1}=\sup S_{1}$ is finite. Clearly, (5.7) yields that $S_{1} \supset\left(-\infty, s_{1}\right)$.

Step 4. $S_{2} \supset\left(-\infty, s_{1}\right)$.

For any $s \in \mathbb{R}$, let $\mathcal{A}(s, \cdot)$ be the fixed point operator in $\mathbb{R}^{N}$ associated to (5.1). Take $s_{2}<$ $s_{1}<s_{3}$. It follows from Lemma 5.1 that there is a $\rho$ such that each possible zero of $I-\mathcal{A}(s, \cdot)$ with $s \in\left[s_{2}, s_{3}\right]$ is $\mathbf{u} \in B_{\rho}$. Furthermore, the Brouwer degree $\operatorname{deg}\left(I-\mathcal{A}(s, \cdot), B_{\rho}, \mathbf{0}\right)$ is well defined and does not depend upon $s \in\left[s_{2}, s_{3}\right]$. Notice that $\mathbf{u}-\mathcal{A}(s, \mathbf{u}) \neq 0$ for all $\mathbf{u} \in \mathbb{R}^{N}$ by using (5.8), which means that $\operatorname{deg}\left(I-\mathcal{A}\left(s_{3}, \cdot\right), B_{\rho}, \mathbf{0}\right)=0$, so that $\operatorname{deg}\left(I-\mathcal{A}\left(s_{2}, \cdot\right), B_{\rho}, \mathbf{0}\right)=0$. By the excision property of the Brouwer degree, $\operatorname{deg}\left(I-\mathcal{A}\left(s_{2}, \cdot\right), B_{\rho}^{\prime}, \mathbf{0}\right)=0$ with $\rho^{\prime}>\rho$. Let $s \in\left(s_{2}, s_{3}\right)$ and $\hat{\mathbf{u}}$ be a solution of (5.1). Then $\hat{\mathbf{u}}$ is a strict upper solution of (5.1) with $s=s_{2}$. Choose $R<\hat{\mathbf{u}}_{L}^{-}$such that $\max _{k \in[2, N-1]} f(k, R, 0)<s_{2}$. Then $R$ is a strict lower solution of (5.1) with $s=s_{2}$. Subsequently, from Theorem 4.2, (5.1) has a solution in $\Omega_{\hat{\mathbf{u}}, R}$ with $s=s_{2}$ and $\operatorname{deg}\left(I-\mathcal{A}\left(s_{2}, \cdot\right), \Omega_{\hat{\mathbf{u}}, R}, \mathbf{0}\right)=-1$. Take $\rho^{\prime}$ large enough, it follows from the additivity property of the Brouwer degree [19] that

$$
\begin{aligned}
\operatorname{deg}\left(I-\tilde{\mathcal{A}}\left(s_{2}, \cdot\right), B_{\rho^{\prime}} \backslash \Omega_{\hat{\mathbf{u}}, R}, \mathbf{0}\right) & =\operatorname{deg}\left(I-\tilde{\mathcal{A}}\left(s_{2}, \cdot\right), B_{\rho^{\prime}}, \mathbf{0}\right)-\operatorname{deg}\left(I-\tilde{\mathcal{A}}\left(s_{2}, \cdot\right), \Omega_{\hat{\mathbf{u}}, R}, \mathbf{0}\right) \\
& =\operatorname{deg}\left(I-\tilde{\mathcal{A}}\left(s_{2}, \cdot\right), \Omega_{\hat{\mathbf{u}}, R}, \mathbf{0}\right)=1 .
\end{aligned}
$$

This implies that (5.1) has the second solution in $B_{\rho^{\prime}} \backslash \Omega_{\hat{\mathbf{u}}, R}$ with $s=s_{2}$.

Step 5. We claim that $s_{1} \in S_{1}$.

Let $\left\{\eta_{j}\right\}$ be a sequence in $\left(-\infty, s_{1}\right)$ satisfying $\lim _{j \rightarrow \infty} \eta_{j}=s_{1}$, and let $\mathbf{u}^{j}$ be a solution of (5.1) with $s=\eta_{j}$ given by Step 3. Then we deduce from Lemma 2.3 that

$$
\mathbf{u}^{j}=\mathcal{A}\left(\eta_{j}, \mathbf{u}^{j}\right)
$$


Applying Lemma 5.1, there exists $\rho>0$ such that $\left\|\mathbf{u}^{j}\right\|<\rho(j \geq 1)$. Since $\mathcal{A}$ is completely continuous, there is a subsequence of $\mathbf{u}^{j}$, relabeling if necessary such that $\mathbf{u}^{j} \rightarrow \mathbf{u} \in \mathbb{R}^{N}$ and satisfies $\mathbf{u}=\mathcal{A}\left(s_{1}, \mathbf{u}\right)$. That is, $\mathbf{u}$ is a solution of (5.1) with $s=s_{1}$.

Last, we shall give a similar result for the following dual Ambrosetti-Prodi condition.

Theorem 5.3 Suppose that a continuous function $f$ satisfies the coercivity condition

$$
f(k, u, v) \rightarrow+\infty \quad \text { if }|u| \rightarrow \infty \text { uniformly for } k \in[2, N-1]_{\mathbb{Z}}, v \in(-a, a),
$$

$h_{1}, h_{N}: \mathbb{R} \rightarrow \mathbb{R}$ are continuous and satisfy the following conditions:

$h_{1}$ is bounded from below, $h_{1}(0)=0, h_{1}(s) \geq 0, s \geq 0$;

$h_{N}$ is bounded from above, $h_{N}(0)=0, h_{N}(s) \leq 0, s \geq 0$.

Then there exists $s_{1} \in \mathbb{R}$ such that problem (5.1) has no solution with $s<s_{1}$, at least one solution with $s=s_{1}$, or at least two solutions with $s>s_{1}$.

Corollary 5.4 Let $g:[2, N-1]_{\mathbb{Z}} \times \mathbb{R}^{2} \rightarrow \mathbb{R}, p:[2, N-1]_{\mathbb{Z}} \times \mathbb{R} \rightarrow \mathbb{R}, h_{1}: \mathbb{R} \rightarrow \mathbb{R}, h_{N}: \mathbb{R} \rightarrow \mathbb{R}$ be continuous. If $g$ is bounded on $[2, N-1]_{\mathbb{Z}} \times \mathbb{R} \times(-a, a) h_{1}, h_{N}$ satisfy (5.3), (5.4) (resp. (5.11), (5.12)) and p satisfies

$$
p(k, s) \rightarrow+\infty \quad(\text { resp. }-\infty) \quad \text { if }|s| \rightarrow \infty \text { uniformly in } k \in[2, N-1]_{\mathbb{Z}}
$$

Then there exists $s_{1} \in \mathbb{R}$ such that the following problem

$$
\begin{aligned}
& \nabla\left(\phi\left(\Delta u_{k}\right)\right)+p\left(k, u_{k}\right)=s+g\left(k, u_{k}, \Delta u_{k}\right), \quad k \in[2, N-1]_{\mathbb{Z}}, \\
& \phi\left(\Delta u_{1}\right)=h_{1}\left(u_{1}\right), \quad \phi\left(\Delta u_{N-1}\right)=h_{N}\left(u_{N}\right)
\end{aligned}
$$

has no solution with $s>s_{1}$ (resp. $\left.s<s_{1}\right)$, at least one solution with $s=s_{1}$, and at least two solutions with $s<s_{1}$ (resp. $s>s_{1}$ ).

Example 5.5 Let $\mathbf{e}=\left(e_{2}, e_{3}, \ldots, e_{N-1}\right) \in \mathbb{R}^{N-2}, m \in \mathbb{R}, n<0$ (resp. $\left.n>0\right), p>0$ and $q \geq 0$. Then there exists $s_{1} \in \mathbb{R}$ such that the problem

$$
\begin{aligned}
& \nabla\left(\phi\left(\Delta u_{k}\right)\right)+m\left|\Delta u_{k}\right|^{q}+n\left|u_{k}\right|^{p}=s+e_{k}, \quad k \in[2, N-1]_{\mathbb{Z}}, \\
& \phi\left(\Delta u_{1}\right)=h_{1}\left(u_{1}\right), \quad \phi\left(\Delta u_{N-1}\right)=h_{N}\left(u_{N}\right)
\end{aligned}
$$

has no solution with $s>s_{1}$ (resp. $s<s_{1}$ ), at least one solution with $s=s_{1}$, and at least two solutions with $s<s_{1}$ (resp. $s>s_{1}$ ), where $h_{1}, h_{N}: \mathbb{R} \rightarrow \mathbb{R}$ satisfy (5.3), (5.4) (resp.(5.11), (5.12)), $\phi(s)=\frac{s}{\sqrt{1-s^{2}}}$.

\section{Acknowledgements}

The authors are very grateful to the anonymous referees for their valuable suggestions. This work is supported by NSFC (No.11626188, No.11671322, No.11501451), Gansu provincial National Science Foundation of China (No.1606RJYA232) and NWNU-LKQN-15-16. 


\section{Competing interests}

The authors confirm that they have read SpringerOpen's guidance on competing interests and have included these in the manuscript. The authors also declare that there is no conflict of interests regarding the publication of this paper.

\section{Authors' contributions}

LY and MR completed the main study, carried out the results of this article and drafted the manuscript, LB checked the proofs and verified the calculation. All the authors read and approved the manuscript.

\section{Author details}

'Department of Mathematics, Northwest Normal University, Lanzhou, 730070, P.R. China. ${ }^{2}$ College of Mathematics and Computer Science, Northwest University for Nationalities, Lanzhou, 730030, P.R. China.

\section{Publisher's Note}

Springer Nature remains neutral with regard to jurisdictional claims in published maps and institutional affiliations.

\section{Received: 4 July 2017 Accepted: 21 September 2017 Published online: 05 October 2017}

\section{References}

1. Bereanu, C, Mawhin, J: Existence and multiplicity results for some nonlinear problems with singular $\phi$-Laplacian. J. Differ. Equ. 243, 536-557 (2007)

2. Bereanu, C, Mawhin, J: Nonhomogeneous boundary value problems for some nonlinear equations with singular $\phi$-Laplacian. J. Math. Anal. Appl. 352, 218-233 (2009)

3. Bereanu, C, Jebelean, P, Mawhin, J: Radial solutions for Neumann problems involving mean curvature operators in Euclidean and Minkowski spaces. Math. Nachr. 283(3), 379-391 (2010)

4. Bereanu, C, Jebelean, P, Torres, PJ: Positive radial solutions for Dirichlet problems with mean curvature operators in Minkowski space. J. Funct. Anal. 264, 270-287 (2013)

5. Coelho, I, Corsato, C, Obersnel, F, Omari, P: Positive solutions of the Dirichlet problem for the one-dimensional Minkowski-curvature equation. Adv. Nonlinear Stud. 12(3), 621-638 (2012)

6. $\mathrm{Ma}, \mathrm{R}, \mathrm{Gao}, \mathrm{H}, \mathrm{Lu}, \mathrm{Y}$ : Global structure of radial positive solutions for a prescribed mean curvature problem in a ball. J. Funct. Anal. 270, 2430-2455 (2016)

7. Bereanu, C, Thompson, HB: Periodic solutions of second order nonlinear difference equations with discrete $\phi$-Laplacian. J. Math. Anal. Appl. 330, 1002-1015 (2007)

8. Bereanu, C, Mawhin, J: Boundary value problems for second-order nonlinear difference equations with discrete $\phi$-Laplacian and singular $\phi$. J. Differ. Equ. Appl. 14(10-11), 1099-1118 (2008)

9. Lu, Y, Ma, R: Existence and multiplicity of solutions of second-order discrete Neumann problem with singular $\phi$-Laplacian operator. Adv. Differ. Equ. 2014, 227 (2014)

10. Agarwal, RP: Difference equations and inequalities. In: Theory, Methods, and Applications, 2nd edn. Monographs and Textbooks in Pure and Applied Mathematics, vol. 228. Dekker, New York (2000)

11. Anderson, DR, Rachunková, I, Tisdell, CC: Solvability of discrete Neumann boundary value problems. J. Math. Anal. Appl. 331, 736-741 (2007)

12. Gao, C: On the linear and nonlinear discrete second-order Neumann boundary value problems. Appl. Math. Comput. $233,62-71(2014)$

13. Ma, R, Gao, C, Lu, Y: Spectrum of discrete second-order Neumann boundary value problems with sign-changing weight. Abstr. Appl. Anal. 2013, Article ID 280508 (2013)

14. Lu, Y: Global structure of positive solutions for second-order discrete Neumann problems involving a superlinear nonlinearity with zeros. Adv. Differ. Equ. 2016, 99 (2016). doi:10.1186/s13662-016-0791-9

15. Kelley, WG, Peterson, AC: Difference equations. In: An Introduction with Applications, 2nd edn. Academic Press, San Diego (2001)

16. Henderson, J, Thompson, HB: Difference equations associated with fully nonlinear boundary value problems for second order ordinary differential equations. J. Differ. Equ. Appl. 7(2), 297-321 (2001)

17. Ehme, J, Eloe, PW, Henderson, J: Upper and lower solution methods for fully nonlinear boundary value problems. J. Differ. Equ. 180, 51-64 (2002)

18. Granas, A, Guenther, R, Lee, J: Nonlinear Boundary Value Problems for Ordinary Differential Equations. Dissertationes Mathematicae, vol. 244. Polish Sci., Warszawa (1985)

19. Deimling, K: Nonlinear Functional Analysis. Springer, Berlin (1988) 